\title{
Sexual Development
}

\section{Erratum}

Sex Dev 2022;16:71

Published online: December 21, 2021

DOI: $10.1159 / 000521500$

In the article "Podocnemis expansa Turtles Hint to a Unifying Explanation for the Evolution of Temperature-Dependent Sex Determination in Long-Lived and Short-Lived Vertebrates" [Sex Dev. 2021;15:23-37, DOI: 10.1159/000515208] by Valenzuela, the female and male lines in Figure $2 \mathrm{~d}$ should be reversed.

The full Figure 2 (with the corrected panel d) is shown here.

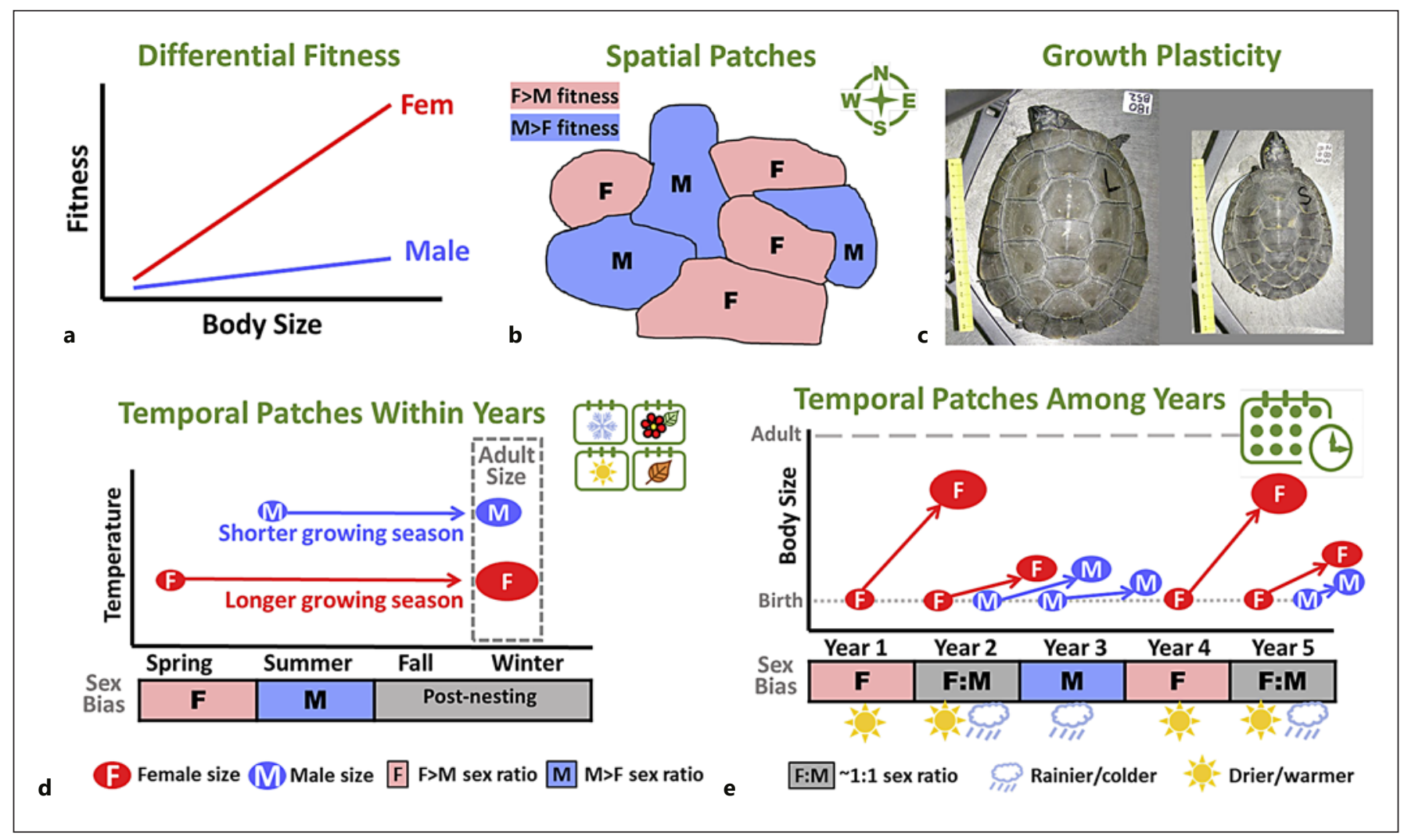

Fig. 2. Fig. 2. Differential fitness hypotheses consistent with the CharnovBull'77 model of adaptive TSD evolution. a Females attain greater fitness gains (e.g., via fecundity) than males as a function of body size. $\mathbf{b}$ Spatially-patchy environments confer greater fitness to one or the other sex in each patch. c Largest and smallest 25-month old $P$. expansa clutch mates exhibiting one of the greatest differences in growth rate during a plasticity study [Ceballos et al., 2014]. See text for details. d Temporally-patchy environments defined by seasonal temperatures within years confer greater fitness to females (i.e., larger body size - fecundity) when colder nest temperatures correlate with a longer growing season. e Temporally-patchy environments defined by inter/supra-annual temperatures (such as those resulting from El Niño or other cyclical climatic events) confer greater fitness to females when warmer temperatures correlate with optimal resource quality/quantity after hatching (note that TSD would be adaptive under this model when high to full feminization occurs during such years). The panel depicts a hypothetical temporal series where optimal conditions repeat after 4 years; for illustration purposes only. 